\title{
Automatic Verification of Advanced Object-Oriented Features: The AutoProof Approach
}

\author{
Julian Tschannen, Carlo A. Furia, Martin Nordio, and Bertrand Meyer \\ Chair of Software Engineering, ETH Zurich, Switzerland \\ \{firstname.lastname\}@inf.ethz.ch
}

\begin{abstract}
Static program verifiers such as Spec\#, Dafny, jStar, and VeriFast define the state of the art in automated functional verification techniques. The next open challenges are to make verification tools usable even by programmers not fluent in formal techniques. This paper discusses some techniques used in AutoProof, a verification tool that translates Eiffel programs to Boogie and uses the Boogie verifier to prove them. In an effort to be usable with real programs, AutoProof fully supports several advanced object-oriented features including polymorphism, inheritance, and function objects. AutoProof also adopts simple strategies to reduce the amount of annotations needed when verifying programs (e.g., frame conditions). The paper illustrates the main features of AutoProof's translation, including some whose implementation is underway, and demonstrates them with examples and a case study.
\end{abstract}

\section{Usable Verification Tools}

It is hard to overstate the importance of tools for software verification: tools have practically demonstrated the impact of general theoretical principles, and they have brought automation into significant parts of the verification process. Program provers, in particular, have matured to the point where they can handle complex properties of real programs. For example, provers based on Hoare semantics - e.g., Spec\# [2] and ESC/Java [5] - support models of the heap to prove properties of object-oriented applications; other tools using separation logic - e.g., jStar 4 and VeriFast [8] - can reason about complex usages of pointers, such as in the visitor, observer, and factory design patterns. The experience gathered so far has also outlined some design principles, which buttress the development on new, improved verification tools; the success of the Spec\# project, for example, has shown the value of using intermediate languages (Boogie [10], in the case of Spec\#) to layer a complex verification process into simpler components, which can then be independently improved and reused across different projects.

The progress of verification tools is manifest, but it is still largely driven by challenge problems and examples. While case studies will remain important, verification tools must now also become more practical and usable by "lay" programmers. In terms of concrete goals, prover tools should support the complete 
semantics of their target programming language; they should require minimal annotational effort besides writing ordinary pre and postconditions of routines (methods); and they should give valuable feedback when verification fails.

The present paper describes some traits of AutoProof, a static verifier for Eiffel programs that makes some progress towards these goals of increased usability. AutoProof translates Eiffel programs annotated with contracts (pre and postconditions, class invariants, intermediate assertions) into Boogie programs. The translation currently handles sophisticated language features such as exception handling and function objects (called agents in Eiffel parlance, and delegates in

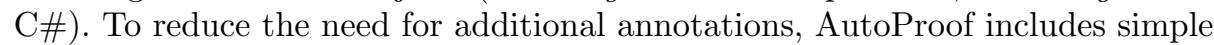
syntactic rules to generate standard frame conditions from postconditions, so that programmers have to write down explicit frame conditions only in the more complex cases.

This paper outlines the translation of Eiffel programs into Boogie, focusing on the most original features such as exception handling (which is peculiarly different in Eiffel, as opposed to other object-oriented languages such as Java and $\mathrm{C \#}$ ) and the generation of simple frame conditions. The translation of more standard constructs is described elsewhere [25]. At the time of writing, AutoProof does not implement the translation of exceptions described in the paper, but its inclusion is underway. The paper also reports a case study where we automatically verify several Eiffel programs, exercising different language features, with AutoProof. AutoProof is part of EVE (Eiffel Verification Environment), the research branch of the EiffelStudio integrated development environment, which integrates several verification techniques and tools. EVE is distributed as free software and available for download at:

$$
\text { http://se.inf.ethz.ch/research/eve/ }
$$

A version of AutoProof is also integrated into CloudStudio 21 web-based multilanguage integrated development environment, available online at:

$$
\text { http://cloudstudio.ethz.ch/, }
$$

Outline. Section 2 introduces the Boogie intermediate language to make the paper self-contained. Section 3 presents the Boogie translation of Eiffel's exception handling primitives; Section 4 describes a translation of conforming inheritance that supports polymorphism; Section 5 discusses a technique to verify function objects. Section 6 sketches other features of the translation, such as the definition of "default" frame conditions; Section 7 illustrates the examples verified in the case study; Section 8 presents the essential related work, and Section 9 outlines future work.

\section{A Short Introduction to Boogie}

Boogie is a language for verification [10, as well as an automated verifier that takes programs written in the Boogie language as input. AutoProof verifies Eiffel programs by translating them into the Boogie language and then by calling 
the Boogie verifier on the translation. The Boogie verifier generates verification conditions for the input program, and supports different prover back-ends (e.g., Z3 and Simplify) to discharge them. For readers unfamiliar with Boogie, this section describes the essential features of the Boogie language used in the rest of the paper.

The Boogie language offers two kinds of constructs: a simple imperative modular programming language - used to translate the source program (Eiffel, in our case) - and a specification language based on first-order logic - used to define specification elements and background logic theories needed to support complex specifications.

Boogie's specification language is a typed first-order logic with arithmetic. The basic types include Booleans (bool) and mathematical (unbounded) integers (int); the type constructors support the definition of derived types. Line 1 in Figure 1 declares a new type person, which Boogie treats as a fresh sort for variables. The specification language supports the definition of global variables and constants, functions (in the sense of mathematical logic), and axioms. Line 2, for example, declares a global constant eve of type person. Lines 3 and 4 declare two functions age and can_vote. Lines 5 and 6 introduce two axioms about the declared items: age is defined as 23 for argument eve; and can_vote is true precisely for persons whose age is greater than or equal to 18 .

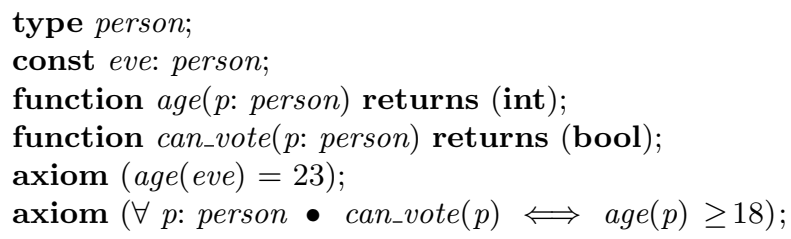

Fig. 1. Some definitions in Boogie's specification language.

Boogie's programming language supports the definition of procedures. Each procedure has a signature, which may include a specification in terms of preconditions (requires), postconditions (ensures), and frame clauses (modifies). The specification clauses contain formulas in Boogie's specification language. Postconditions, in addition, supports the usage of the old keyword to evaluate expressions in the state before a procedure was called. Modifies clauses, instead, define a procedure's frame, that is the set of global variables the procedure may modify. Pre- and postconditions may be marked as free, which prevents the generation of proof obligations based on them: a free assertion is assumed to hold whenever convenient, but need not be checked when required.

Procedure implementations use standard imperative constructs (assignments, conditionals, loops, jumps, and procedure calls) with the usual semantics. To write nondeterministic programs, Boogie's programming language includes the havoc command, which assigns a nondeterministically chosen value to its argu- 
ment variables. To constrain the effects of havoc and to express intermediate verification conditions, Boogie's programming language also offers assert and assume statements. Both take an arbitrary formula $F$ as argument. The program state of every execution reaching an assert $F$ must satisfy $F$; otherwise, verification fails. Conversely, the verification process can assume that $F$ holds of the program state whenever an execution reaches assume $F$, which "shapes" the nondeterministic behavior when convenient.

Figure 2 shows the specification and implementation of a procedure vote to cast a vote, demonstrating Boogie syntax.

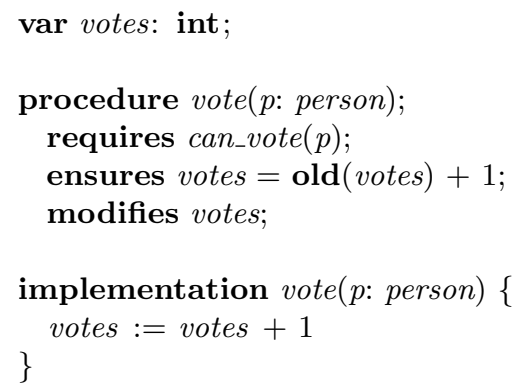

Fig. 2. A Boogie procedure vote: specification and implementation.

\section{Exceptions}

Eiffel's exception handling mechanism is different than most object-oriented programming languages such as C\# and Java ${ }^{1}$ This section presents Eiffel's mechanism (Section 3.1), discusses how to annotate exceptions (Section 3.2), and describes the translation of Eiffel's exceptions to Boogie (Section 3.3) with the help of an example (Section 3.4).

\subsection{How Eiffel Exceptions Work}

Eiffel exception handlers are specific to each routine, where they occupy an optional rescue clause, which follows the routine body (do). A routine's rescue clause is ignored whenever the routine body executes normally. If, instead, executing the routine body triggers an exception, control is transferred to the rescue clause for exception handling. The exception handler will try to restore the object state to a condition where the routine can execute normally. To this

\footnotetext{
${ }^{1}$ In related work, we have formalized the semantics of Java exceptions [16] and com-
} pared it against Eiffel's [17. 
end, the body can run more than once, according to the value of an implicit variable Retry, local to each routine: when the execution of the handler terminates, if Retry has value True the routine body is run again, otherwise Retry is False and the pending exception propagates to the rescue clause of the caller routine.

Figure 3 illustrates the Eiffel exception mechanism with an example. The routine attempt_transmission tries to transmit a message by calling unsafe_transmit; if the latter routine terminates normally, attempt_transmission also terminates normally without executing the rescue clause. On the contrary, an exception triggered by unsafe_transmit transfers control to the rescue clause, which reexecutes the body for max_attempts times; if all the attempts fail to execute successfully, the attribute (field) failed is set and the exception propagates.

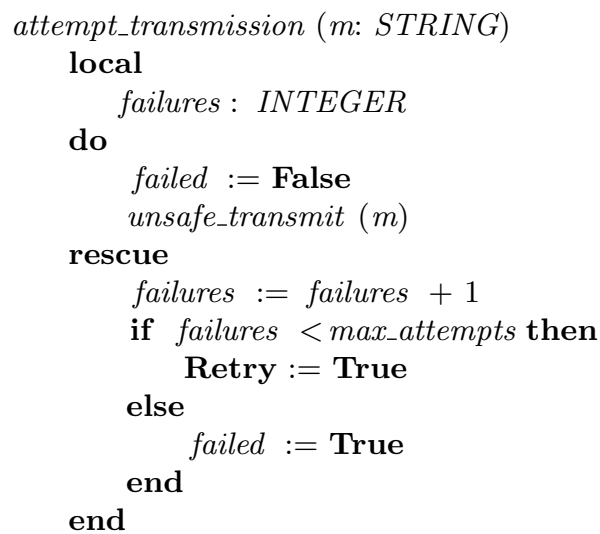

Fig. 3. An Eiffel routine with exception handler.

\subsection{Specifying Exceptions}

The postcondition of a routine with rescue clause specifies the program state both after normal termination and when an exception is triggered. The two post-states are in general different, hence we introduce a global Boolean variable Exc $V$, which is True if and only if the routine has triggered an exception. Using this auxiliary variable, specifying postconditions of routines with exception handlers is straightforward. For example, the postcondition of routine attempt_transmission in Figure 3 says that failed is False if and only if the routine executes normally:

attempt_transmission $(m: S T R I N G)$

ensure

Exc $V$ implies failed 
not ExcV implies not failed

The example also shows that the execution of a rescue clause behaves as a loop: a routine $r$ with exception handler $r$ do $s_{1}$ rescue $s_{2}$ end behaves as the loop that first executes $s_{1}$ unconditionally, and then repeats $s_{2} ; s_{1}$ until $s_{1}$ triggers no exceptions or Retry is False after the execution of $s_{2}$ (in the latter case, $s_{1}$ is not executed anymore). To reason about such implicit loops, we introduce a rescue invariant 20122]; the rescue invariant holds after the first execution of $s_{1}$ and after each execution of $s_{2} ; s_{1}$. A suitable rescue invariant of routine attempt_transmission is:

rescue invariant

not ExcV implies not failed

( failures < max_attempts) implies not failed

\subsection{Eiffel Exceptions in Boogie}

The auxiliary variable $E x c V$ becomes a global variable in Boogie, so that every assertion can reference it. The translation also introduces an additional precondition $E x c V=$ false for every translated routine, because normal calls cannot occur when exceptions are pending, and adds ExcV to the modifies clause of every procedure. Then, a routine with body $s_{1}$ and rescue clause $s_{2}$ becomes in Boogie:

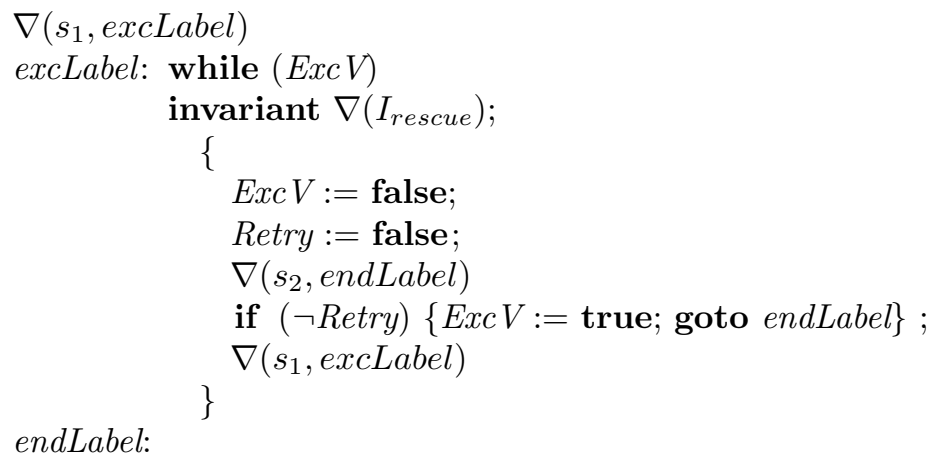

where $\nabla(s, l)$ denotes the Boogie translation $\nabla(s)$ of the instruction $s$, followed by a jump to label $l$ if $s$ triggers an exception:

$$
\nabla(s, l)= \begin{cases}\nabla\left(s^{\prime}, l\right) ; \nabla\left(s^{\prime \prime}, l\right) & \text { if } s \text { is the compound } s^{\prime} ; s^{\prime \prime} \\ \nabla(s) ; \text { if }(\text { ExcV }) \text { \{goto } l ;\} & \text { otherwise }\end{cases}
$$

Therefore, when the body $s_{1}$ triggers an exception, $\operatorname{Exc} V$ is set and the execution enters the rescue loop. On the other hand, an exception that occurs in the body of $s_{2}$ jumps out of the loop and to the end of the routine.

The exception handling semantics is only superficially similar to having control-flow breaking instructions such as break and continue - available in languages other than Eiffel - inside standard loops: the program locations where the 
control flow diverts in case of exception are implicit, hence the translation has to supply a conditional jump after every instruction that might trigger an exception. This complicates the semantics of the source code, and correspondingly the verification of Boogie code translating routines with exception handling.

\subsection{An Example of Exception Handling in Boogie}

Figure 4 shows the translation of the example in Figure 3 . To simplify the presentation, Figure 4 renders the attributes max_attempts, failed, and transmitted (set by unsafe_transmit) as variables rather than locations in a heap map. The loop in lines $24 \sqrt{38}$ maps the loop induced by the rescue clause, and its invariant (lines 25 and 26 ) is the rescue invariant.

\section{Inheritance and Polymorphism}

The redefinition of a routine $r$ in a descendant class can strengthen $r$ 's original postcondition by adding an ensure then clause, which conjoins the postcondition in the precursor. The example in Figure 5 illustrates a difficulty occurring when reasoning about postcondition strengthening in the presence of polymorphic types. The deferred (abstract) class EXP models nonnegative integer expressions and provides a routine eval to evaluate the value of an expression object; even if eval does not have an implementation in EXP, its postcondition specifies that the evaluation always yields a nonnegative value stored in attribute last_value, which is set as side effect (see Section 6.1). Classes CONST and $P L U S$ respectively specialize EXP to represent integer (nonnegative) constants and addition. Class ROOT is a client of the other classes, and its main routine attaches an object of subclass CONST to a reference with static type EXP, thus exploiting polymorphism. Similar issues occur when a descendant class weakens a some routine $r$ 's precondition with an require else clause.

The verification goal is proving that, after the invocation e.eval (in class $R O O T$ ), eval's postcondition in class CONST holds, which subsumes the check statement in the caller. Reasoning about the invocation only based on the static type EXP of the target $e$ guarantees the postcondition last_value $\geq 0$, which is however too weak to establish that last_value is exactly 5 .

Other approaches, such as Müller's [15, have targeted these issues in the context of Hoare logics, but they usually are unsupported by automatic program verifiers. In particular, with the Boogie translation of polymorphic assignment implemented in Spec\#, we can verify the assertion check e.last_value $=5$ end in class ROOT only if eval is declared pure; eval is, however, not pure. The Spec\# methodology selects the pre and postconditions according to static types for nonpure routines: the call e. eval only establishes $e$. last_value $\geq 0$, not the stronger e. last_value $=5$ that follows from $e$ 's dynamic type CONST, unless an explicit cast redefines the type CONST. The rest of the section describes the solution implemented in AutoProof, which handles contracts of redefined routines. 


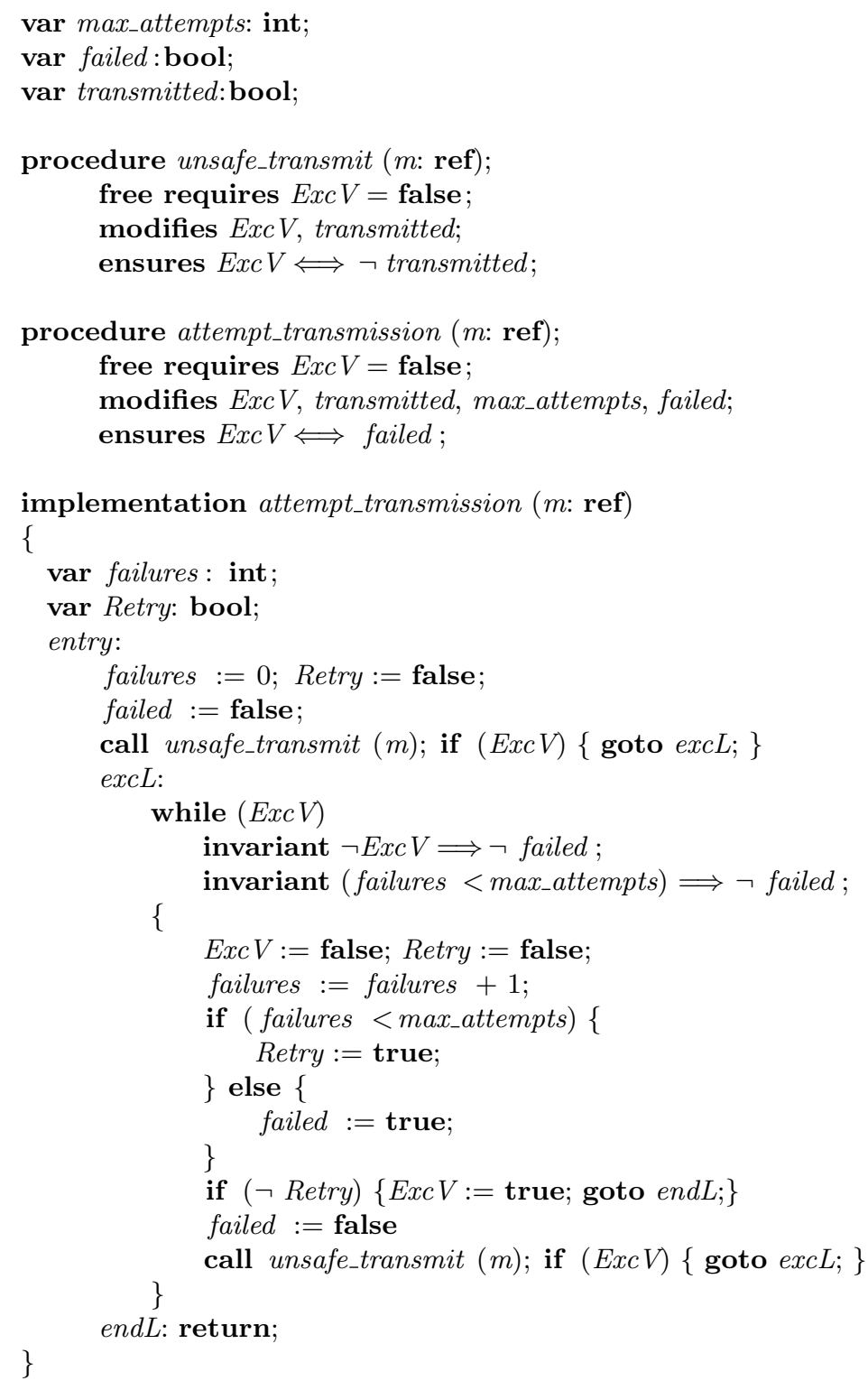

Fig. 4. Boogie translation of the Eiffel routine in Figure 3 


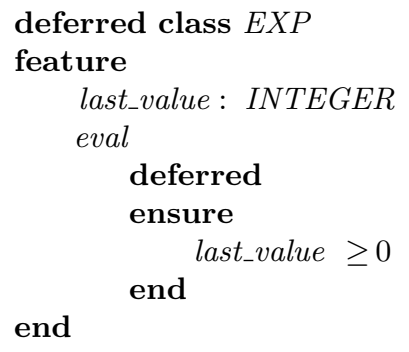

Fig. 5. Nonnegative integer expressions.

\subsection{Polymorphism in Boogie}

The Boogie translation implemented in AutoProof can handle polymorphism appropriately even for non-pure routines; it is based on a methodology for agents [19] and on a methodology for pure routines 312 . The rest of the section discusses how to translate postconditions and preconditions of redefined routines in a way that accommodates polymorphism, while still supporting modular reasoning.

Postconditions. The translation of the postcondition of a routine $r$ of class $X$ with result type $T$ (if any) relies on an auxiliary function post.X.r:

function post.X.r (h1, h2: Heap Type; c: ref; res: T) returns (bool);

which predicates over two heaps (the pre and post-states in $r$ 's postcondition), a reference $c$ to the current object, and the result res. $r$ 's postcondition in Boogie 
references the function post.X.r, and includes the translation $\nabla_{\text {post }}(X . r)$ of $r$ 's postcondition clause syntactically declared in class $X 2^{2}$

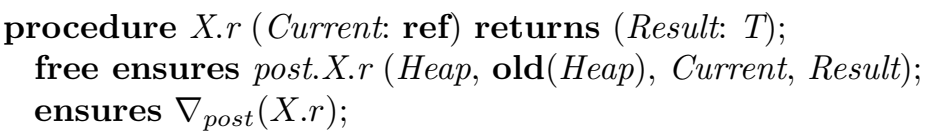

post.X. $r$ is a free ensures, hence it is ignored when proving $r$ 's implementation and is only necessary to reason about usages of $r$.

The function post.X.r holds only for the type $X$; for each class $Y$ which is a descendant of $X$ (and for $X$ itself), an axiom links $r$ 's postcondition in $X$ to $r$ 's strengthened postcondition in $Y$ :

$$
\begin{aligned}
& \underset{\text { axiom }}{ }(\forall h 1, h 2: \text { Heap Type; } c \text { : ref; } r: T \bullet \\
& \left.\$ \text { type }(c)<: Y \Longrightarrow\left(\text { post.X.r }(h 1, h 2, c, r) \Longrightarrow \nabla_{\text {post }}(Y . r)\right)\right) ;
\end{aligned}
$$

The function $\$$ type returns the type of a given reference; hence the postcondition predicate post.X.r implies an actual postcondition $\nabla_{\text {post }}(Y . r)$ according to $c$ 's dynamic type.

In addition, for each redefinition of $r$ in a descendant class $Z$, the translation defines a fresh Boogie procedure Z.r with corresponding postcondition predicate post.Z.r and axioms for all of $Z$ 's descendants.

Preconditions. Eiffel also supports weakening of preconditions. Therefore, the precondition of a routine can also depend on the dynamic type. We use a similar translation as for the postcondition. Given a routine $r$ of type $X$, a precondition predicate is generated and used in the signature of the generated Boogie procedure:

function pre.X.r(h: HeapType; c: ref) returns (bool);

which predicates over one heap and a reference $c$ to the current object. $r$ 's precondition in Boogie references the function pre.X.r, and it includes the translation $\nabla_{\text {pre }}(X . r)$ of $r$ 's precondition originally declared in class $X$ :

procedure X.r (Current: ref) returns (Result: T);

requires pre.X.r(Heap, Current);

free requires $\nabla_{\text {pre }}(X . r)$

Conversely to the postcondition, establishing $r$ 's precondition is a responsibility of callers of $r$; clients have to establish the precondition determined by the dynamic type - captured by the function pre.X.r-, whereas the precondition originally given in $X$ is given as a free requires and is only used to prove $r$ 's implementation.

$$
\begin{aligned}
& \text { axiom }(\forall h \text { : HeapType; } c: \text { ref } \bullet \\
& \left.\quad h[c, \text { type }]<: Y \Longrightarrow\left(\nabla_{\text {pre }}(Y . r) \Longrightarrow \text { pre.X.r }(h, c)\right)\right) ;
\end{aligned}
$$

To establish pre.X.r, it is enough to establish any of the clauses $\nabla_{\text {pre }}(Y . r)$.

\footnotetext{
$\overline{2}$ The translation differs for calls to Precursor (super in Java and base in C\#).
} 


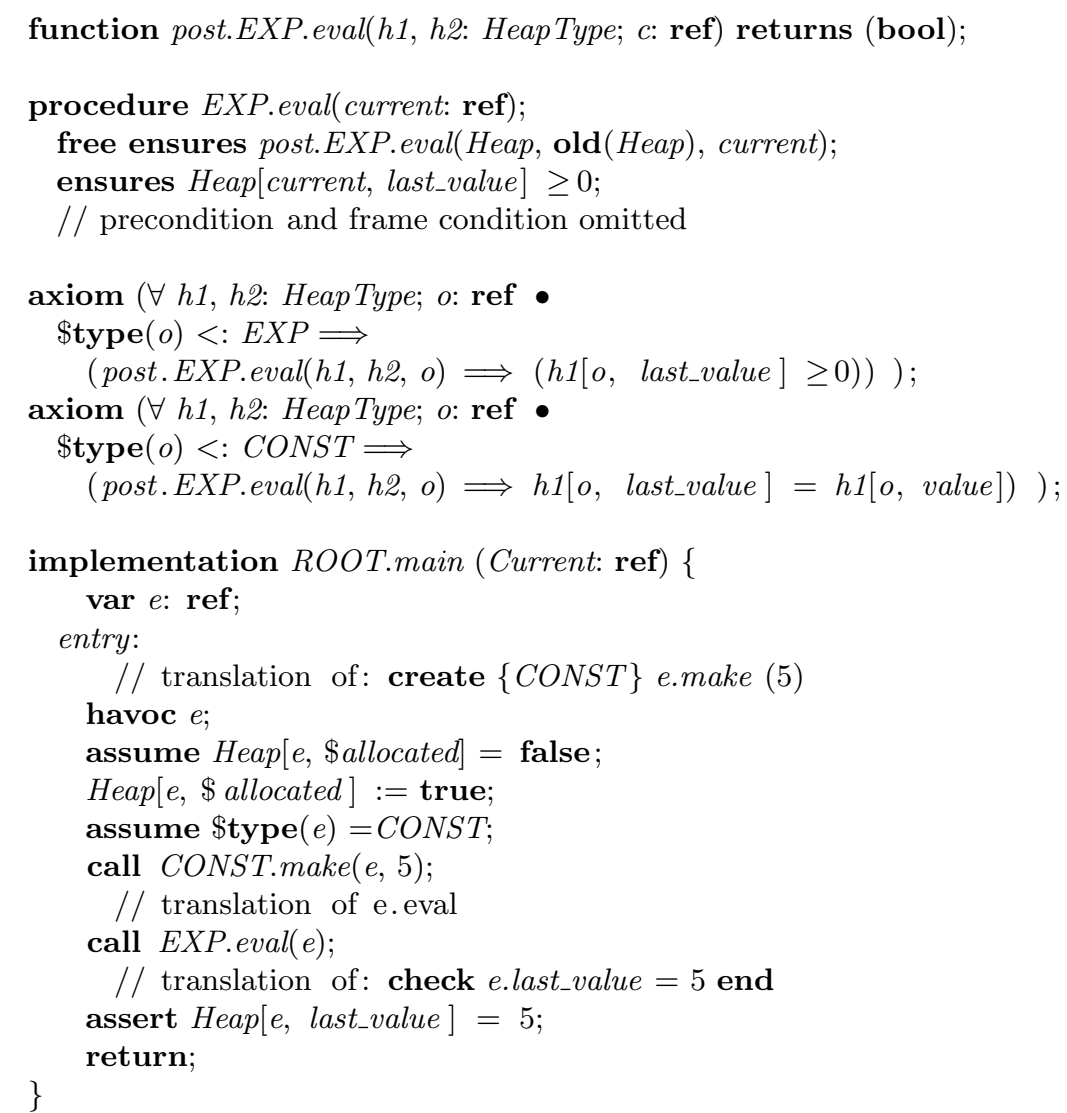

Fig. 6. Boogie translation of the Eiffel classes in Figure 5. 


\subsection{An Example of Polymorphism with Postconditions}

Figure 6 shows the essential parts of the Boogie translation of the example in Figure 5. The translation of routine eval in lines 3-6 references the function post.EXP.eval; the axioms in lines 8-13 link such function to $r$ 's postcondition in EXP (lines 8-10) and to the additional postcondition introduced in CONST for the same routine (lines 11-13). The rest of the figure shows the translation of the client class ROOT.

\section{$5 \quad$ Agents (Function Objects)}

Eiffel programs can use agents - called function objects, closures, or delegates in other languages. Supporting agents in verification poses a number of challenges; Section 5.1 illustrates the main such challenges while succinctly describing Eiffel agents' syntax and semantics with an example. Then, Section 5.2 discusses how to specify agents; Sections 5.3 and 5.4 describe the translation of agents implemented in AutoProof; and Section 5.5 presents how the translation handles framing. This section is based on our previous work [19].

\subsection{An Example Using Agents}

We illustrate the challenges of verifying agents using an example by Leavens et al. 9] adapted for Eiffel. Consider a class FORMATTER that collects routines operating on paragraphs. The class includes, among others, the routine align_left $(p: P A R A G R A P H)$ that aligns to the left the text in paragraph $p$, passed as argument. For some details of its implementation that we need not delve into, the routine requires that the paragraph is not already aligned to the left; if executed correctly, it ensures that $p$ is changed so that it is left aligned. Assuming class paragraph includes a (pure) routine left_aligned that returns true when called on paragraphs that are left aligned, we can write align_left 's specification in FORMATTER as:

class FORMATTER

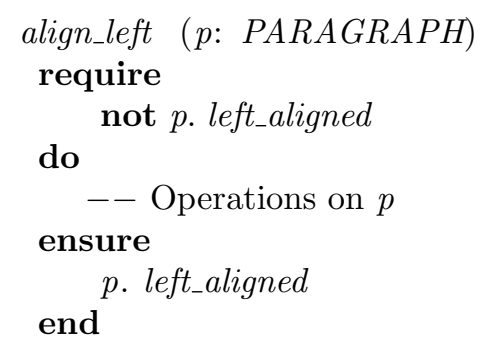

end

Class FORMATTER includes other routines that operate on paragraphs, such as align_right, justify, and add_margin, each with its proper specification. 
Clients of class FORMATTER can apply any given routine of the class to objects of type PARAGRAPH. To this end, class PARAGRAPH offers a routine format that takes a generic routine of class FORMATTER - wrapped in an agent - and applies it to the current object of class PARAGRAPH. This is how format can be written in Eiffel:

class PARAGRAPH

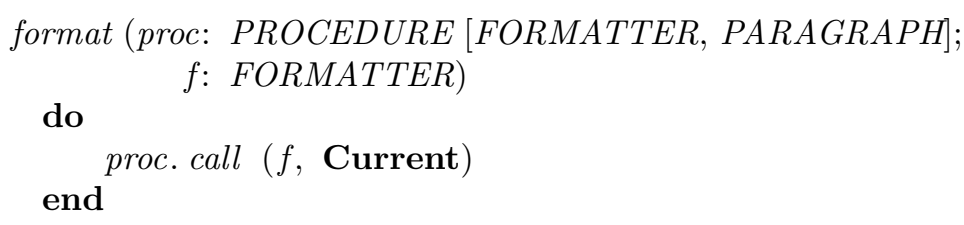

The first argument has type PROCEDURE [FORMATTER, PARAGRAPH]; this denotes an agent, whose target class is FORMATTER, with an argument of class PARAGRAPH ${ }^{3}$ in other words, if $f$ has type FORMATTER, $p$ has type paragraph, and proc wraps some routine $m$ in FORMATTER, f. $m(p)$ is a typecorrect call. Agent invocation uses the different syntax shown in the example above: proc. call ( $f$, Current) calls the routine wrapped by proc on the target $f$, passing it the Current object of class PARAGRAPH as argument.

This showed how agents are invoked. Let us now demonstrate agent creation in Eiffel with a routine apply_align_left that calls align_left on a paragraph through format:

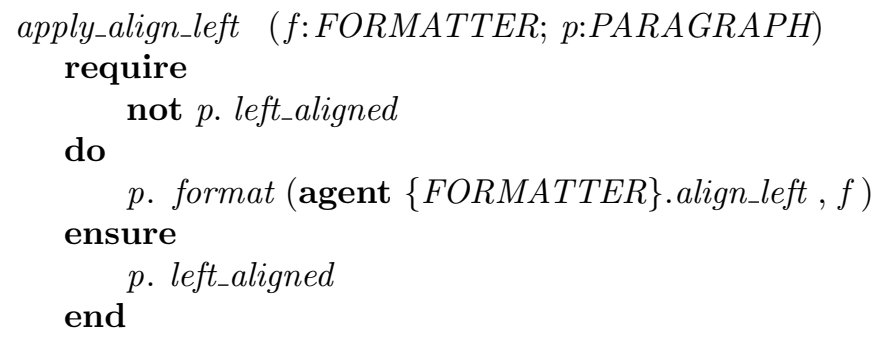

The expression agent $\{F O R M A T T E R\}$.align_left denotes an agent that wraps routine align_left of class FORMATTER ${ }^{4}$ This agent definition does not bind the wrapped routine to a specific target or to an argument $p$; therefore, we call it an agent with open arguments. In contrast, the expression agent $f$. align_left $(p)$ denotes an agent with closed arguments: the target is bound to $f$ and the argument to $p$.

Verifying apply_align_left boils down to proving the correctness of the call p.format. This, in turn, requires: 1) having a specification of format; 2) being

\footnotetext{
${ }^{3}$ For illustration purposes, we slightly simplify Eiffel's syntax and we limit ourselves to the case of single-argument routines. The generalization is humdrum.

${ }^{4}$ The keyword agent is necessary to disambiguate between a function object wrapping align_left and the invocation of align_left .
} 
able to discharge format's precondition by means of apply_align_left 's and to establish apply_align_left 's postcondition from format's; 3) specifying format's frame and deduce apply_align_left's frame from it. In conformance with the general verification style of AutoProof (and Boogie), we should handle these problems modularly: format's specification and correctness proof should be independent of how format is used by clients (and, in particular, which agents it receives as argument). These are the challenges of verifying function objects: Section 5.2 discusses how to write specifications for agents; Sections 5.3 and 5.4 shows how AutoProof uses agents; and Section 5.5 deals with agent framing.

\subsection{Specifying Agents}

Agents are abstract placeholders for routines; the actual routine attached to an argument of type agent is, in general, known only dynamically. In fact, the purpose of agents is providing a generic container of routines; the specification of agents must conform to the same level of abstraction.

In Eiffel, variables referring to agents all belong to class ROUTINE or some of its descendants (such as PROCEDURE in the FORMATTER example). Specification of agents can then use the functions precondition and postcondition of the class, which return a Boolean expression respectively corresponding to the require and ensure clause of the actual routine wrapped by an agent. In the running example, we can use these functions to specify format parametrically with respect to its argument proc of agent type:

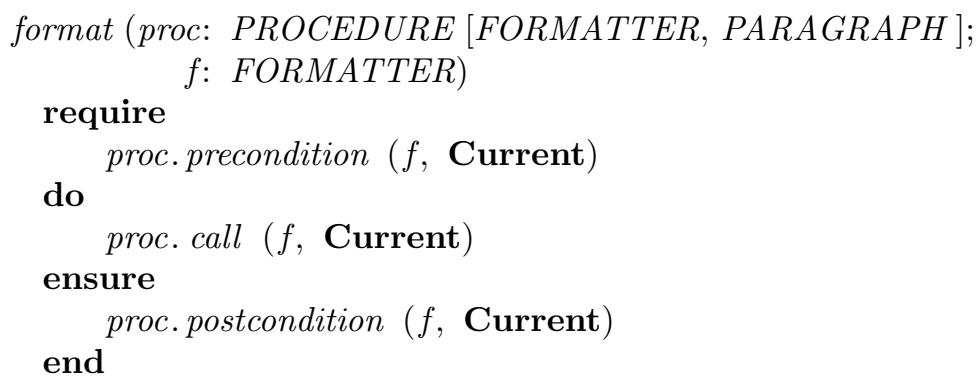

Using the same mechanism, we can also specify the generic pre- and postcondition of call in class ROUTINE:

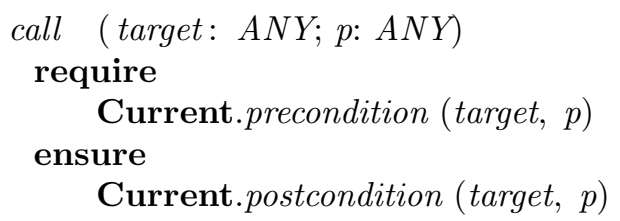

AutoProof's translation uses such generic specifications to reason modularly about the correctness of programs using agents, as described in the following subsections. 


\subsection{Agents in Boogie: Open Arguments}

This section describes the basics of AutoProof's translation of Eiffel agents to Boogie; the presentation focuses on agents with open target and arguments. Section 5.4 outlines how AutoProof deals with closed arguments.

Translating agent specification. The translation introduces two uninterpreted Boolean functions to model the precondition and postcondition of agents:

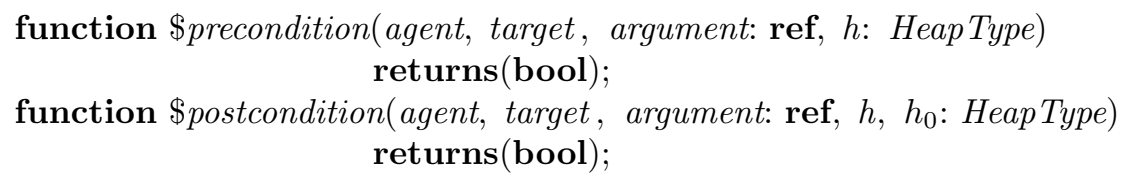

The function arguments represent references to objects for the agent, its target, and its arguments, plus a copy of the heap $h$ and, for postconditions, the "old" heap $h_{0}$ before the agent was invoked.

Translating agent invocation. Consider three reference variables $a, t$, and $p$, respectively representing an agent, a target object, and an argument object in the Boogie translation. The agent invocation $a$. call $(t, p)$ is translated as follows:

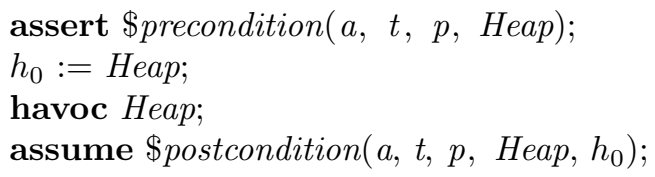

That is, verify that $a$ 's precondition holds of the current heap; save the heap to $h_{0}$ and nondeterministically change its content; assume that the new heap satisfies $a$ 's postcondition.

Translating agent creation. When an agent is created, we must bind the placeholders \$precondition and \$postcondition to the actual pre- and postcondition of the routine wrapped by the agent, so that they can be used in the correctness proofs to reason about agent usages. If $a$ represents in Boogie a reference attached to an agent created as agent $p r$ from some routine $p r$, the following two assumptions bind pr's pre- and postcondition (represented in Boogie by functions pre.pr and post.pr):

$$
\begin{aligned}
& \text { assume } \forall t, p: \text { ref, } h_{1}: \text { HeapType } \bullet \\
& \text { \$precondition }\left(a, t, p, h_{1}\right)=\operatorname{pre.pr}\left(t, p, h_{1}\right) ; \\
& \text { assume } \forall t, p: \text { ref, } h_{1}, h_{2}: \text { HeapType } \bullet \\
& \text { \$postcondition }\left(a, t, p, h_{1}, h_{2}\right)=\text { post.pr }\left(t, p, h_{1}, h_{2}\right) ;
\end{aligned}
$$

\subsection{Agents in Boogie: Closed Arguments}

This section discusses the translation of agents with either closed target or closed argument. The generalization to closed target and argument is straightforward. 
Translating agent specification. The translation introduces two uninterpreted Boolean functions with different signature to model the precondition and postcondition of agents with closed arguments:

function $\$$ precondition $_{1}$ (agent, arg: ref, $h$ : HeapType) returns(bool);

function $\$$ postcondition $_{1}$ (agent, arg: ref, $h, h_{0}$ : Heap Type) returns(bool);

The argument arg represents either a reference to the open target (if the agent's argument is closed) or a reference to the open argument (if the agent's target is closed). The other function arguments are as in Section 5.1

Translating agent invocation. Consider two reference variables $a$ and $p$, respectively representing an agent (with closed target) and an argument object in the Boogie translation. The agent invocation $a$. call $(p)$ is translated as:

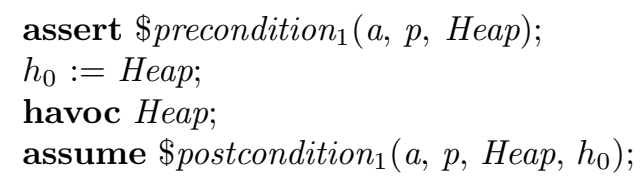

The same translation works if $a$ has closed argument and open target, and $p$ translates a reference to a target object.

Translating agent creation. If $a$ represent in Boogie a reference attached to an agent created as agent $u$.pr from some routine $p r$ and target $u$, the translation generates the following two assumptions:

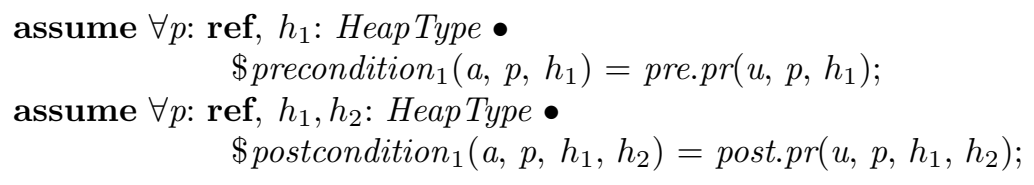

Similarly, if $b$ represent in Boogie a reference attached to an agent created as agent $\operatorname{pr}(v)$ from some routine $p r$ and argument $v$, the translation generates the following two assumptions:

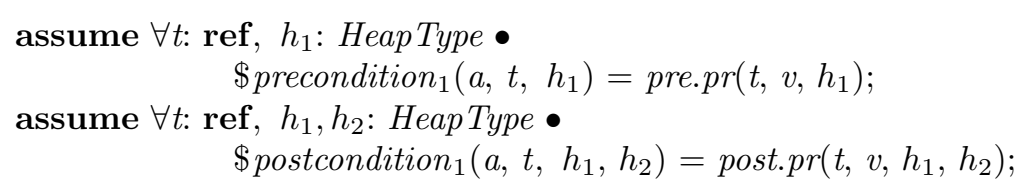

\subsection{Framing of Agents}

Eiffel does not offer explicit support to specify frame conditions, that is the portion of the heap that a routine may modify. In principle, this is not necessary in many cases, because we can express changed and unchanged entities in postconditions. Following this intuition, AutoProof offers a simple mechanism that can generate frame conditions in the simplest cases; we describe it in Section 6.1. 
When proving programs with agents, however, the kind of annotations necessary to express frame conditions in postconditions become cumbersome. This is essentially due to the fact that the frame of an agent depends, in general, also on its dynamically attached target and arguments, which need to be used in its frame specification. Therefore, we introduce modify clauses in Eiffel to specify agent framing. In practice, these are implemented as note annotations, which does not require changing the parser and guarantees compatibility with any version of the language.

Specifying frame conditions. Following what we did for pre- and postconditions in Section 5.2, we equip class ROUTINE with a function modifies that returns the list of locations modified by the actual routine wrapped by an agent 5 modify clauses can then include both lists of object references that may be directly modified by a routine and lists of calls to modifies functions. The rest of the presentation considers agents with open target and arguments; the modifications necessary to deal with closed arguments are described in a technical report [18. In the running example, we can use this function to specify format's frame as being whatever proc may modify when called on target $f$ and argument Current:

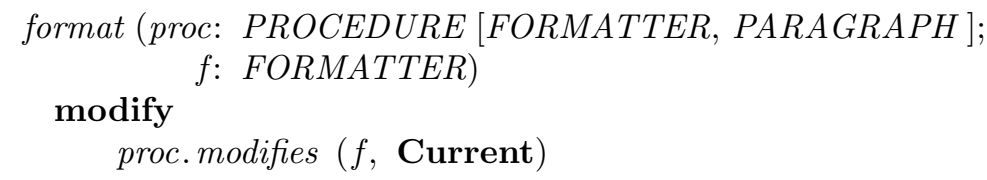

Translating modify clauses. The translation introduces an uninterpreted function \$modify: function $\$$ modify(agent, target, arg: ref, $h$ : Heap Type,
obj: ref, fid: FieldId) returns(bool);

The function arguments represent references to objects for an agent, its target, and its arguments, a copy of the heap, as well as an additional generic reference $o b j$ to an object and the identifier fid of one of its attributes (fields). Intuitively, if $\$ \operatorname{modify}(a, t, p, h, o, f)$ is true, the call $a$. call $(t, p)$ may modify the value of $o . f$ in the heap $h$.

AutoProof translates a generic Eiffel modify clause of some routine $r$ :

modify $a_{1}$.modifies $\left(t_{1}, p_{1}\right), \ldots, a_{m}$.modifies $\left(t_{m}, p_{m}\right), o_{1}, \ldots, o_{n}$

into a postcondition clause of $r$ 's translation to Boogie:

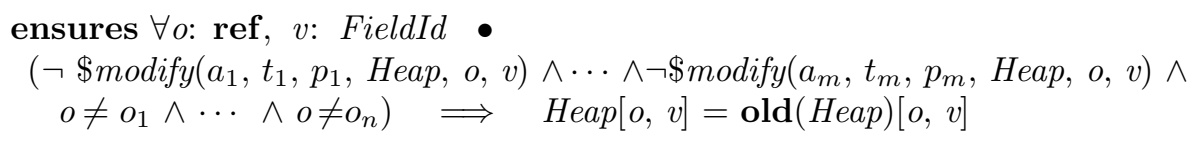

${ }^{5}$ Since modifies is only used in modify clauses, which are not part of the Eiffel language, we need not provide any actual implementation of modifies. 
That is, all objects and attributes not explicitly mentioned in the modify clause $\left(o_{1}, \ldots, o_{n}\right)$ and not modified by the agents (modifies) are certainly not changed by routine $r$. For example, the format's modify clause translates to:

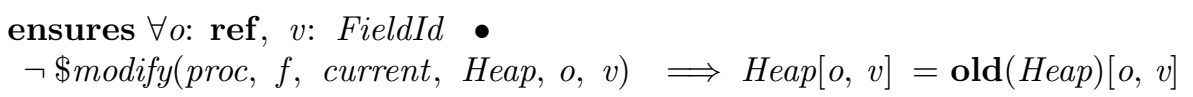

Frame conditions from agent creation. Finally, when an agent is created, we bind the placeholder \$modify to the actual locations modified by the routine wrapped by the agent. Similarly to what we showed in Section 5.3 for pre- and postconditions, if $a$ represents in Boogie a reference attached to an agent created as agent $p r$ from some routine $p r$, the translation generates the assumption:

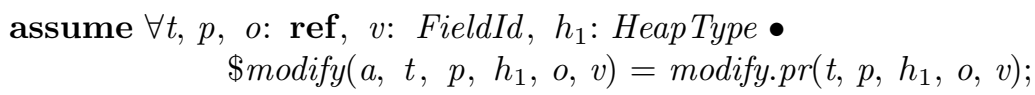

where modify.pr represents the encoding of $p r$ 's frame in Boogie (whose details are straightforward).

Current limitations. The translation of agent framing currently implemented in AutoProof does not support the specification of fine-grained frame disjointness properties. In particular, it is not possible to specify that the locations modified by two agents are disjoint. This is often necessary when reasoning about agents working on composite data structures. The Eiffel library class LIST, for example, offers a routine do_all that takes an agent passed as argument and applies it to every element of the list. Reasoning about do_all requires to distinguish between when the agent is applied to different elements of the list; the simple convention of modify clauses, however, does not offer this level of granularity. In our technical report [18, we suggest a mechanism to express such non-interference properties; its implementation in AutoProof is part of future work.

\section{Other Features}

This section briefly presents other features of the Eiffel-to-Boogie translation.

\subsection{Default Frame Conditions}

Frame conditions are necessary to reason modularly about heap-manipulating programs, but they are also an additional annotational burden for programmers. In several simple cases, however, the frame conditions are straightforward and can be inferred syntactically from the postconditions. For a routine $r$, let $\bmod _{r}$ denote the set of attributes mentioned in $r$ 's postcondition; $\bmod _{r}$ is a set of (reference, attribute) pairs. The translation of Eiffel to Boogie implemented in AutoProof assumes that every attribute in $\bmod _{r}$ may be modified (that is, $\bmod _{r}$ is $r$ 's frame), whereas every other location in the heap is not modified. Since every non-pure routine already includes the whole Heap map in its modifies clause, the frame condition becomes the postcondition clause: 


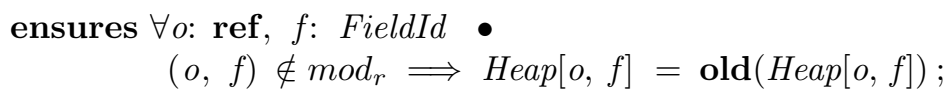

To ensure soundness in the presence of inheritance, the translation always uses the postcondition of the original routine definition to infer the frame of the routine's redefinitions.

The frame conditions inferred by AutoProof work well for routines whose postconditions only mention attributes of primitive type. For routines that manipulate more complex data, such as arrays or lists, the default frame conditions are too coarse-grained; hence programmers have to supplement them with more detailed annotations. Extending the support for automatically generated frame conditions is part of future work.

\subsection{Routines Used in Contracts Pure by Default}

The translation of routines marked as pure generates the frame condition ensures Heap $=\operatorname{old}($ Heap $)$ which specifies that the heap is unchanged. AutoProof implicitly assumes that every routine used in contracts is pure, and the translation reflects this assumption and checks its validity. While the Eiffel language does not require routines used in contract to be pure, it is a natural assumption which holds in practice most of the times, because the behavior of a program should not rely on whether contracts are evaluated or not. Therefore, including this assumption simplifies the annotational burden and makes using AutoProof easier in practice.

\section{Case Study}

This section presents the results of a case study applying AutoProof to the verification of the 11 programs listed in Table 1. For each example, the table reports its name, its size in number of classes and lines of code, the length (in lines of code) of the translation to Boogie, the time taken by Boogie to verify successfully the example, and the kind of Eiffel features mostly exercised by the example.

Example 1 is a set of routines presented in Meyer's book [14] when describing Eiffel exceptions; Example 2 is a set of classes part of the EiffelStudio compiler runtime. To verify them, we extended the original contracts with postconditions to express the behavior when exceptions are triggered, and with rescue invariants (Section 3.2) ${ }^{6}$ The most difficult part of verifying these example was inventing rescue invariants. Even when the examples are simple, the rescue invariants may be subtle, because they have to include clauses both for normal and for exceptional termination.

Examples 3-5 target polymorphism in verification. The Expression example is described in Section 4. The Sequence example models integer sequences with

\footnotetext{
${ }^{6}$ As the implementation in AutoProof of translation of exceptions is currently underway, these two examples were translated by hand.
} 


\begin{tabular}{|c|c|c|c|c|c|}
\hline EXAMPLE NAME & ClaAsses & LOC EIFFEL & LOC BoogIE & Time $[\mathrm{S}]$ & Feature \\
\hline 1. Textbook OOSC2 & 1 & 106 & 481 & 2.33 & Exceptions \\
\hline 2. Runtime ISE & 4 & 203 & 561 & 2.32 & Exceptions \\
\hline 3. Expression & $\dot{4}$ & $1 \dot{3} 4^{\circ}$ & $75 \dot{2}$ & 2.11 & Inheritance \\
\hline 4. Sequence & 5 & 195 & 976 & 2.28 & Inheritance \\
\hline 5. Command & 4 & 99 & 714 & 2.14 & Inheritance \\
\hline 6. Formatter & 3 & 120 & 761 & 2.23 & Agents \\
\hline 7. Archiver & 4 & 121 & 915 & 2.07 & Agents \\
\hline 8. Calculator & 3 & 245 & 1426 & 9.73 & Agents \\
\hline 9. Cell / Recell & 3 & $1 \dot{5} \dot{4}$ & 905 & $2.0 \dot{0}$ & General \\
\hline 10. Counter & 2 & 97 & 683 & 2.02 & General \\
\hline 11. Account & 2 & 120 & 669 & 2.04 & General \\
\hline Total & 35 & 1594 & 8843 & 31.36 & \\
\hline
\end{tabular}

Table 1. Examples automatically verified with AutoProof

the deferred classes SEQUENCE, MONOTONE_SEQUENCE, and STRICT SEQUENCE, and their effective descendants ARITHMETIC_SEQUENCE, and FIBONACCI_SEQUENCE. The Command example implements the command design pattern [7] with a deferred class COMMAND and effective descendants that augment the postcondition of COMMAND's deferred routine execute. The encoding of inheritance described in Section 4 is accurate but it also significantly increases the size of the Boogie translation and correspondingly the time needed to handle it. Since a translation that takes dynamic types into account is not always necessary, we have introduced an option to have AutoProof translating contracts solely based on the static type of references. This speeds up verification in the most common cases, while still having the option to use the more complex encoding when necessary.

Examples 6-8 use agents and are the same examples as in [19. The Formatter example illustrates the specification of functions taking agents as arguments; the Archiver example uses an agent with closed arguments; the Calculator example implements the command design pattern using agents rather than subclasses.

Examples 9-11 combine multiple features: a cell class that stores integer values; a counter that can be increased and decreased; a bank account class with clients. These examples demonstrate other features of the translation, such as the usage of default frame conditions.

The source code of the examples is available at http://se.ethz.ch/people/ tschannen/boogie2011_examples.zip. The experiments ran on a Windows 7 machine with a $2.71 \mathrm{GHz}$ dual core Intel Pentium processor and 2GB of RAM.

\section{Related Work}

Tools such as ESC/Java [5] and Spec\# 20 have made considerable progress towards practical and automated functional verification. Spec\# is an extension of C\# with syntax to express preconditions, postconditions, class invariants, and 
non-null types. Spec\# is also a verification environment that verifies Spec\# programs by translating them to Boogie - also developed within the same project. Spec\# works on significant examples, but it does not support every feature of C\# (for example, delegates are not handled, and exceptions can only be checked at runtime). Spec\# includes annotations to specify frame conditions, which make proofs easier but at the price of an additional annotational burden for developers. To ease the annotational overhead, Spec\# adds a default frame condition that includes all attributes of the target object. This solution has the advantage that the frame can change with routine redefinitions to include attributes introduced in the subclasses. AutoProof follows a different approach and tries to rely on standard annotations whenever possible, which impacts on the programs that can be verified automatically.

Spec\# has shown the advantages of using an intermediate language for verification. Other tools such as Dafny [11] and Chalice [13], and techniques based on Region Logic 1, follow this approach, and they also rely on Boogie as intermediate language and verification back-end, in the same way as AutoProof does.

Separation logic 23] is an extension of Hoare logic with connectives that define separation between regions of the heap, which provides an elegant approach to reasoning about programs with mutable data structures. Verification environments based on separation logic - such as jStar 4 and VeriFast [8 — can verify advanced features such as usages of the visitor, observer, and factory design patterns. On the other hand, writing separation logic annotations requires considerably more expertise than using standard contracts embedded in the programming language; this makes tools based on separation logic more challenging to use by practitioners.

In our previous work [26, we have presented a verification tool that integrates static proofs and dynamic testing techniques.

\section{Future Work}

AutoProof is a component of EVE, the Eiffel Verification Environment, which combines different verification tools to exploit their synergies and provide a uniform and enhanced usage experience, with the ultimate goal of getting closer to the idea of "verification as a matter of course".

Future work will extend AutoProof and improve its integration with other verification tools in EVE. In particular, the design of a translation supporting the expressive model-based contracts 24 is currently underway. Other aspects for improvements are a better inference mechanism for frame conditions and intermediate assertions (e.g., loop invariants [6]); a support for interactive prover as an alternative to Boogie for the harder proofs; and a combination of AutoProof with the separation logic prover also part of EVE [28].

Acknowledgments. This work has been partially funded by the SNF grant LSAT (200020-134974) and by the Hasler foundation on related projects. A 
preliminary version of this work has been presented at the First International Workshop on Intermediate Verification Languages (Boogie'11), held in Wrocław, Poland, in August 2011 and is available as technical report [27].

\section{References}

1. A. Banerjee, D. A. Naumann, and S. Rosenberg. Regional logic for local reasoning about global invariants. In In European Conference on Object Oriented Programming, ECOOP. Springer-Verlag, 2008.

2. M. Barnett, K. R. M. Leino, and W. Schulte. The Spec\# Programming System: An Overview. In CASSIS, volume 3362 of LNCS, pages 49-69. Springer, 2004.

3. A. Darvas and K. R. M. Leino. Practical reasoning about invocations and implementations of pure methods. In FASE, LNCS. Springer-Verlag, 2007.

4. D. Distefano and M. J. Parkinson. jStar: Towards Practical Verification for Java. In Proceedings of OOPSLA, pages 213-226, 2008.

5. C. Flanagan, K. R. M. Leino, M. Lillibridge, G. Nelson, J. B. Saxe, and R. Stata. Extended static checking for Java. In PLDI, pages 234-245. ACM, 2002.

6. C. A. Furia and B. Meyer. Inferring loop invariants using postconditions. In Fields of Logic and Computation, volume 6300 of Lecture Notes in Computer Science, pages 277-300. Springer, 2010.

7. E. Gamma, R. Helm, R. Johnson, and J. Vlissides. Design Patterns: Elements of Reusable Object-Oriented Software. Addison Wesley, 1994.

8. B. Jacobs, J. Smans, and F. Piessens. A quick tour of the VeriFast program verifier. In Proceedings of APLAS 2010, 2010.

9. G. T. Leavens, K. R. M. Leino, and P. Müller. Specification and verification challenges for sequential object-oriented programs. Formal Aspects of Computing, 19(2):159-189, 2007.

10. K. R. M. Leino. This is Boogie 2. Technical report, Microsoft Research, 2008.

11. K. R. M. Leino. Dafny: an automatic program verifier for functional correctness. In Proceedings of the 16th international conference on Logic for programming, artificial intelligence, and reasoning, LPAR-16, pages 348-370. Springer-Verlag, 2010.

12. K. R. M. Leino and P. Müller. Verification of equivalent-results methods. In ESOP, volume 4960 of $L N C S$, pages 307-321. Springer-Verlag, 2008.

13. K. R. M. Leino and P. Müller. A basis for verifying multi-threaded programs. In Proceedings of the 18th European Symposium on Programming Languages and Systems, ESOP '09, pages 378-393. Springer-Verlag, 2009.

14. B. Meyer. Object-Oriented Software Construction. Prentice Hall, second edition, 1997.

15. P. Müller. Modular Specification and Verification of Object-Oriented Programs, volume 2262 of $L N C S$. Springer-Verlag, 2002.

16. P. Müller and M. Nordio. Proof-transforming compilation of programs with abrupt termination. In SAVCBS '07: Proceedings of the 2007 conference on Specification and verification of component-based systems, pages 39-46, 2007.

17. M. Nordio. Proofs and Proof Transformations for Object-Oriented Programs. PhD thesis, ETH Zurich, Switzerland, 2009.

18. M. Nordio, C. Calcagno, B. Meyer, and P. Müller. Reasoning about Function Objects. Technical Report 615, ETH Zurich, 2008.

19. M. Nordio, C. Calcagno, B. Meyer, P. Müller, and J. Tschannen. Reasoning about Function Objects. In Proceedings of TOOLS-EUROPE, LNCS, pages 79-96. Springer, 2010. 
20. M. Nordio, C. Calcagno, P. Müller, and B. Meyer. A Sound and Complete Program Logic for Eiffel. In M. Oriol, editor, TOOLS-EUROPE, volume 33 of Lecture Notes in Business and Information Processing, pages 195-214, 2009.

21. M. Nordio, H.-C. Estler, C. A. Furia, and B. Meyer. Collaborative software development on the web, 2011. arXiv:1105.0768v3.

22. M. Nordio, P. Müller, and B. Meyer. Proof-Transforming Compilation of Eiffel Programs. In R. Paige and B. Meyer, editors, TOOLS-EUROPE, Lecture Notes in Business and Information Processing, pages 316-335. Springer-Verlag, 2008.

23. P. W. O'Hearn, H. Yang, and J. C. Reynolds. Separation and information hiding. In POPL '04, pages 268-280, 2004.

24. N. Polikarpova, C. A. Furia, and B. Meyer. Specifying reusable components. In Proceedings of VSTTE'10, volume 6217 of Lecture Notes in Computer Science, pages 127-141. Springer, 2010.

25. J. Tschannen. Automatic verification of Eiffel programs. Master's thesis, Chair of Software Engineering, ETH Zurich, 2009.

26. J. Tschannen, C. A. Furia, M. Nordio, and B. Meyer. Usable verification of objectoriented programs by combining static and dynamic techniques. In Proceedings of the 9th International Conference on Software Engineering and Formal Methods (SEFM '11), LNCS, pages 382-398. Springer, 2011.

27. J. Tschannen, C. A. Furia, M. Nordio, and B. Meyer. Verifying Eiffel programs with Boogie. In First International Workshop on Intermediate Verification Languages (BOOGIE), 2011. Available at http://arxiv.org/abs/1106.4700

28. S. van Staden, C. Calcagno, and B. Meyer. Verifying executable object-oriented specifications with separation logic. In Proceedings of ECOOP'10, volume 6183 of Lecture Notes in Computer Science, pages 151-174. Springer, 2010. 